\title{
Altered Cardiac Troponin T In Vitro Function in the Presence of a Mutation Implicated in Familial Hypertrophic Cardiomyopathy
}

\author{
David Lin, ${ }^{\star}$ Alyona Bobkova, ${ }^{\ddagger}$ Earl Homsher, ${ }^{\ddagger}$ and Larry S. Tobacman* \\ ${ }^{*}$ Departments of Internal Medicine and Biochemistry, The University of Iowa, Iowa City, Iowa 52242; and ${ }^{\ddagger}$ Department of Physiology, \\ School of Medicine, University of California at Los Angeles, Los Angeles, California 90024
}

\begin{abstract}
Familial hypertrophic cardiomyopathy (HCM) can be caused by dominant missense mutations in cardiac troponin T (TnT), $\alpha$-tropomyosin, C-protein, or cardiac myosin heavy chain genes. The myosin mutations are known to impair function, but any functional consequences of the TnT mutations are unknown. This report describes the in vitro function of troponin containing an Ile91Asn mutation in rat cardiac TnT, corresponding to the HCM-causing Ile79Asn mutation in man. Mutant and wild-type TnT cDNAs were expressed in bacteria and the proteins purified and reconstituted with the other troponin subunits. The mutation had no effect on troponin's affinity for tropomyosin, troponininduced binding of tropomyosin to actin, cooperative binding of myosin subfragment 1 to the thin filament, $\mathrm{Ca}^{2+}$-sensitive regulation of thin filament-myosin subfragment 1 ATPase activity, or the $\mathrm{Ca}^{2+}$ concentration dependence of this regulation. However, the mutation resulted in $50 \%$ faster thin filament movement over a surface coated with heavy meromyosin in in vitro motility assays. The increased sliding speed suggests an unexpected role for the amino terminal region of TnT in which this mutation occurs. The relationship between this faster motility and altered cardiac contraction in patients with HCM is discussed. (J. Clin. Invest. 1996. 97:2842-2848.) Key words: troponin • cardiomyopathy $\bullet$ tropomyosin $\bullet$ heart $\bullet$ genetics
\end{abstract}

\section{Introduction}

Familial hypertrophic cardiomyopathy $(\mathrm{HCM})^{1}$ can be caused by dominant missense mutations in any one of four genes encoding sarcomeric proteins: $\beta$-cardiac myosin heavy chain, cardiac C-protein (a thick filament accessory protein), $\alpha$-tropomyosin, or cardiac troponin T (TnT) (1-4). The first identified source of HCM was a missense mutation in the myosin heavy chain gene (1), resulting in an Arg403Gln mutation, and a striking defect in the function of the purified myosin. Filament sliding speed is reduced by $80 \%$ in an in vitro motility assay (5). Similarly, the actin-myosin MgATPase rate is greatly di-

Address correspondence to Larry S. Tobacman, Department of Internal Medicine, University of Iowa, Iowa City, IA 52242. Phone: 319-356-3703; FAX: 319-356-7893.

Received for publication 16 November 1995 and accepted in revised form 2 April 1996.

1. Abbreviations used in this paper: HCM, hypertrophic cardiomyopathy; S-1, subfragment 1; TnC, troponin C; TnI, troponin I; TnT, troponin $\mathrm{T}$; wt TnT, recombinant embryonic rat cardiac troponin $\mathrm{T}$.

J. Clin. Invest.

(C) The American Society for Clinical Investigation, Inc.

0021-9738/96/06/2842/07 \$2.00

Volume 97, Number 12, June 1996, 2842-2848 minished by this myosin mutation (6). At least 29 different missense mutations in the myosin heavy chain gene have been found in HCM kindreds, and the functional defects attributable to the mutations can generally be understood in terms of functional regions in the myosin subfragment 1 (S-1) crystal structure (7). The various mutations are associated with variably severe defects in myosin function (8), which may correlate with the severity and penetrance of the disease.

In contrast to the actively investigated mutations in the myosin heavy chain, the recently identified HCM-causing mutations in cardiac TnT are as yet unstudied. Also, the detailed relationship between the TnT mutations and cardiomyopathy is more problematic than for myosin because the three dimensional structure of TnT is unknown. Numerous structure-function studies of TnT have been reported, providing valuable characterization of the properties of TnT fragments and regions (see reviews in 9-11), but the functions of individual residues have not been investigated. Furthermore, two of the eight reported HCM-associated TnT mutations are within an $\mathrm{NH}_{2}$-terminal region that can be deleted with little loss of troponin function (12-15). To address how one of these TnT mutations, Ile79Asn, causes HCM, this report characterizes the functional properties of the homologous mutation in recombinant rat cardiac TnT. In qualitative agreement with previous structure-function studies of TnT, the present results indicate that wild-type TnT and this mutant TnT have few functional differences in vitro. However, the mutation produces one functional change that may be important in explaining the human phenotype: thin filaments containing the mutant TnT exhibit 50\% faster sliding speed in an in vitro motility assay.

\section{Methods}

Cloning strategy. Rat cardiac embryonic TnT cDNA in plasmid expression vector pET3D (16) was subcloned into the BamHI and SpHI sites of the high copy plasmid pSP72. The TnT sequence was altered using oligonucleotide-directed mutagenisis (Clontech, Palo Alto, CA). Nucleotides ATC, encoding isoleucine, were changed to AAT, encoding asparagine. This Ile91Asn missense mutation replaces the normal nonpolar Ile with a polar Asn residue. The complete nucleotide sequences of recombinant embryonic rat cardiac troponin $\mathrm{T}$ (wt TnT) and mutant TnT cDNA were confirmed by a combination of radioactive dideoxynucleotide chain termination (17) and automated fluorescence sequencing (University of Iowa DNA Core, Iowa City, Iowa) methods. The wt and mutant TnT cDNAs were removed from pSP72 by digestion with BamHI and SpHI and inserted back into the same sites on the expression vector $\mathrm{pET} 3 \mathrm{D}$.

Protein expression and purification. DE3 cells were transformed with pET3D encoding either the wt or the mutant TnT sequence. Yields of $\sim 8 \mathrm{mg}$ of TnT per liter of culture were obtained by inoculating $500 \mathrm{ml}$ of Luria broth containing $100 \mathrm{mg} /$ liter ampicillin with a single colony from a fresh transformation. (A much lower expression was observed for the adult cardiac TnT isoform.) The cells were grown overnight at $37^{\circ} \mathrm{C}$, and then $0.4 \mathrm{mM}$ isopropyl- $\beta$-D-thiogalactopyranoside (IPTG) was added for $4 \mathrm{~h}$. Pelleted cells were resus- 
pended in buffer containing $25 \mathrm{mM}$ Tris- $\mathrm{HCl}$ ( $\mathrm{pH} 7.5$ ), $20 \%$ sucrose, $1 \mathrm{mM}$ EDTA, $5 \mu \mathrm{g} / \mathrm{ml}$ TPCK, $5 \mu \mathrm{g} / \mathrm{ml}$ TLCK, $0.3 \mathrm{mM}$ PMSF, and $0.2 \mathrm{M} \mathrm{NaCl}$. The cells were incubated on ice for $1 \mathrm{~h}$ and then stored at $-70^{\circ} \mathrm{C}$ overnight. The cells were thawed and sufficient $5 \mathrm{M} \mathrm{NaCl}$ was added to give $1 \mathrm{M} \mathrm{NaCl}$ final concentration. The cells were then lysed with a probe sonicator. After a $1 \mathrm{~h}$ centrifugation at 46,000 rpm, at $4^{\circ} \mathrm{C}$, the pellet was reextracted with a similar buffer that contained $5 \mathrm{M}$ urea and $0.1 \%$ Triton X100. The extraction step was repeated to dissolve as much of the relatively insoluble TnT as possible. TnT's presence and purity was judged by SDS-PAGE. The TnTs were purified by two successive DE52 (Whatman Inc., Clifton, NJ) column chromatography steps.

Bovine cardiac troponin C ( $\mathrm{TnC})$, troponin I ( TnI), and tropomyosin and rabbit skeletal muscle actin and myosin S-1 were obtained as previously described (18-20).

Troponin reconstitution. Ternary troponin complexes containing mutant or wt TnT, bovine cardiac TnC, and bovine cardiac TnI were obtained by mixing the subunits in 1:1:1 stoichiometry under denaturing conditions, and then sequentially dialyzing to facilitate gradual subunit association (18). To eliminate uncomplexed subunits, the reconstituted troponins were concentrated and applied to a Sephadex G100 column.

MgATPase assay. The rate of $\gamma-\left[{ }^{32} \mathrm{P}\right] \mathrm{ATP}$ hydrolysis was measured by extraction into isobutanol/benzene of $\left.{ }^{32} \mathrm{P}\right] \mathrm{Pi}$ molybdate (21). Six aliquots were extracted over $10 \mathrm{~min}$ and rates were calculated. Free $\mathrm{Ca}^{2+}$ concentrations were calculated using overall $\mathrm{Ca}^{2+}$-1, 2-bis-(2-amino-s-bromo-phenoxy)ethane-N, $\mathrm{N}, \mathrm{N}^{1}, \mathrm{~N}^{1}$ tetraacetic acid (dibromo-BAPTA) and $\mathrm{Mg}^{2+}$-dibromo-BAPTA dissociation constants of $140 \mathrm{nM}$ and $2.8 \mathrm{mM}$, respectively $(22,23)$.

Troponin binding to pyrene-labeled tropomyosin. Fluorescence measurements were performed on stirred, water-jacketed samples using a SLM-8000C spectrofluorometer at $25^{\circ} \mathrm{C}$. Data was obtained with excitation and emission wavelengths of $340 \mathrm{~nm}$ and $405 \mathrm{~nm}$, respectively. The effects of protein dilution $(\sim 10 \%)$ were corrected in the calculations. The conditions studied were: $0.1 \mu \mathrm{M}$ pyrene-labeled tropomyosin, $10 \mathrm{mM}$ Tris- $\mathrm{HCl}(\mathrm{pH} 7.5), 60 \mathrm{mM} \mathrm{KCl}, 3 \mathrm{mM} \mathrm{MgCl}{ }_{2}$, $0.6 \mathrm{mM}$ EGTA, and $0.1 \mathrm{mM}$ dithiothreitol.

Labeling of tropomyosin. Bovine cardiac tropomyosin was stoichiometrically labeled at Cys190 by incubation of the reduced, denatured protein with $\mathrm{N}$-(1-pyrene)-iodoacetamide (Molecular Probes, Inc., Eugene, OR) $(24)$ or $\left[{ }^{3} \mathrm{H}\right]$ iodoacetic acid $\left(1.8 \times 10^{8} \mathrm{cpm} / \mu \mathrm{mol}\right.$; Amersham Corp., Arlington HTS, IL) (12).

Light-scattering assay. Myosin S-1 binding to the thin filament complex was measured by light-scattering intensity at $400 \mathrm{~nm}$ using a SLM $8000 \mathrm{C}$ spectrofluorometer at $25^{\circ} \mathrm{C}$. Myosin S-1 titrations were carried out as the sample was mixed by a microstirrer under the following conditions: $0.5 \mu \mathrm{M}$ bovine cardiac tropomyosin, $3.5 \mu \mathrm{M}$ rabbit skeletal muscle actin, $0.55 \mu \mathrm{M}$ wt or mutant reconstituted troponin, $2 \mathrm{mM}$ ADP, $0.1 \mathrm{mM}$ EGTA, $5 \mathrm{mM} \mathrm{MgCl}_{2}, 150 \mathrm{mM} \mathrm{NaCl}, 20 \mathrm{mM}$ Hepes (pH 7.5), $1 \mathrm{mM}$ dithiothreitol, and $0.02 \% \mathrm{NaN}_{3}$.

Binding of troponin-tropomyosin to F-actin. Binding of radiolabeled tropomyosin to F-actin was determined by cosedimentation in a TLA100 rotor (Beckman Instruments, Inc., Fullerton, CA), spun at $35,000 \mathrm{rpm}$ for $20 \mathrm{~min}$ after $30 \mathrm{~min}$ of presedimentation incubation at the desired temperature. High ionic strength $(300 \mathrm{mM} \mathrm{KCl})$ was employed to prevent troponin-tropomyosin polymerization (12). Conditions were: $10 \mathrm{mM}$ Tris- $\mathrm{HCl}$ (pH7.5), $3 \mathrm{mM} \mathrm{MgCl}_{2}, 300 \mathrm{mM} \mathrm{KCl}, 0.5$ mM EGTA, $5 \mu \mathrm{M}$ F-actin, $0.6 \mu \mathrm{M}$ tropomyosin, and either $25^{\circ} \mathrm{C}$ or $37^{\circ} \mathrm{C}$.

In vitro motility experiments. The movement of rhodamine phalloidin-labeled thin filaments over rabbit fast skeletal muscle-heavy meromyosin-coated coverslips was observed by video epifluorescence microscopy and analyzed as previously described $(25,26)$. Thin filaments were reconstituted with $2 \mu \mathrm{M}$ labeled F-actin, $0.5 \mu \mathrm{M}$ troponin, and $0.5 \mu \mathrm{M}$ tropomyosin. The labeled thin filaments were diluted to an actin concentration of $20-40 \mathrm{nM}$, and $100 \mathrm{nM}$ troponin and tropomyosin were added to maintain actin-troponin-tropomyosin binding $(24,26)$. The experiments were performed at an ionic strength of $100 \mathrm{mM}$ in the presence of $0.5 \%$ methyl cellulose. The temperature of the assay was thermostated at $25^{\circ} \mathrm{C}$. The pCa was var- ied by using $2 \mathrm{mM}$ EGTA as previously described (26). Movement of the centroid of each filament in the field was analyzed at 1-10 frames/s (depending upon sliding speed), and those filaments moving at a consistent speed (i.e., the standard deviation of the frame to frame speed was $<0.5 \times$ the mean speed) were identified. Most of the filaments moved consistently or not at all (26). Under each condition, the movement of more than 100 filaments was analyzed. Reproducibility of the in vitro motility assay has been established using replicate samples of the same protein under identical conditions. In Homsher et al. (26), five motility assays of unregulated thin filaments yielded mean velocities between 4.7 and $5.1 \mu \mathrm{m} / \mathrm{s}$ with the overall mean velocity of the five replicate assays at $5.0 \pm 0.2 \mu \mathrm{m} / \mathrm{s} \mathrm{SD}$.

\section{Results}

Effect of I91N mutation on affinity of reconstituted troponin for tropomyosin. Reconstituted troponin complexes were formed by combining bovine cardiac TnC and TnI with either wt or mutant I91N TnT. These purified troponin complexes are shown in Fig. 1. The rat TnTs are higher in molecular weight than bovine cardiac TnT, consistent with their respective amino acid sequences (27-29).

TnT is the tropomyosin-binding subunit of troponin, so the possible effect of the mutation on troponin-tropomyosin binding was investigated, using tropomyosin labeled with pyrene at cys190. Isoleucine 91 in rat cardiac embryonic TnT is located near the region most clearly shown to bind tightly to tropomyosin (11, 14, 30-32). Addition of troponin caused an increase in tropomyosin pyrene monomer fluorescence (Fig. 2), regardless of whether the TnT in the reconstituted system was bovine, wt, or rat I91N mutant, which corresponds to the I79N mutation causing HCM in man. The concentration dependence of the fluorescence change can be used to determine tropomyosin affinities for reconstituted troponin containing wt or mutant TnT. The representative curves demonstrate that the affinities of pyrene-labeled tropomyosin for wt and mutant troponin were indistinguishable in the presence of EGTA: $1.2 \pm 0.1 \times$ $10^{6} \mathrm{M}^{-1}$ (SEM) for troponin reconstituted with wt TnT and $1.2 \pm 0.2 \times 10^{6} \mathrm{M}^{-1}$ for troponin reconstituted with mutant TnT. Similarly, the mutation did not alter the affinity of troponin for tropomyosin in presence of $\mathrm{Ca}^{2+}$ (affinities of both forms of troponin for tropomyosin were $1.1 \pm 0.2 \times 10^{6} \mathrm{M}^{-1}$ ). These results with reconstituted, chimeric bovine/rat troponins

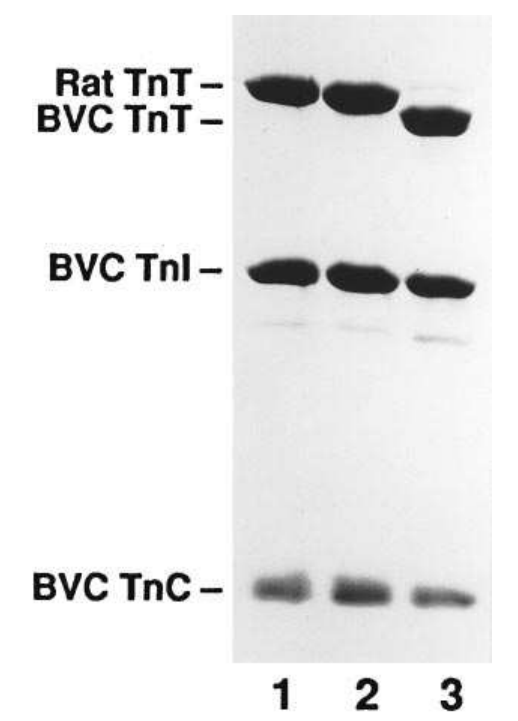

Figure 1. SDS-PAGE of various troponins. Equal amounts of each troponin were loaded on the gel. Lane 1 , reconstituted mutant troponin, comprised of bovine cardiac TnC, bovine cardiac $\mathrm{TnI}$, and $\mathrm{I} 91 \mathrm{~N}$ rat cardiac TnT. Lane 2, reconstituted wt troponin, comprised of bovine cardiac TnC, bovine cardiac TnI, and wt rat cardiac TnT. Lane 3, bovine cardiac whole troponin, isolated as a ternary complex. 


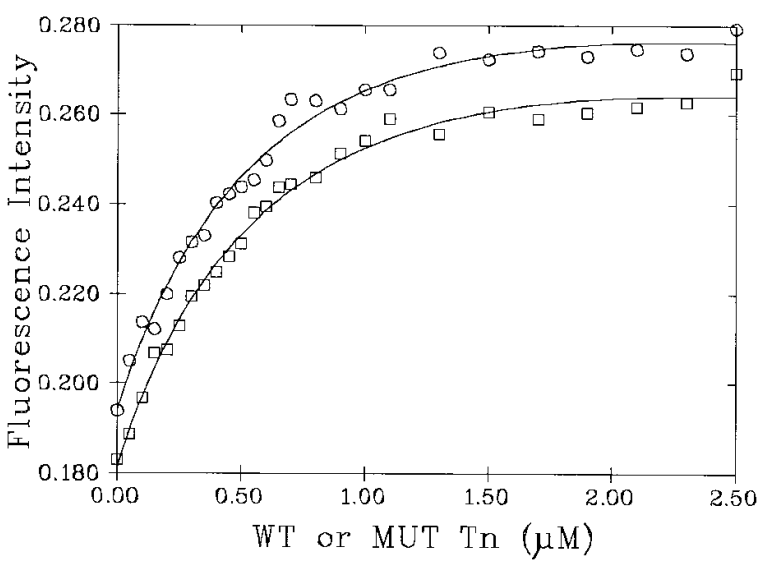

Figure 2. Fluorescence measurement of the affinity of tropomyosin for wt or mutant reconstituted troponin. The pyrene monomer fluorescence intensity (excitation, $340 \mathrm{~nm}$; emission, $405 \mathrm{~nm}$ ) of fluorophore-modified tropomyosin was monitored as increasing concentration of wt or mutant $(\mathrm{I} 91 \mathrm{~N})$ reconstituted troponin were added. Conditions: $25^{\circ} \mathrm{C}, 0.1 \mu \mathrm{M}$ pyrene-modified tropomyosin, $10 \mathrm{mM}$ Tris$\mathrm{HCl}$ (pH 7.5), $60 \mathrm{mM} \mathrm{KCl,} 3 \mathrm{mM} \mathrm{MgCl} 2,0.6 \mathrm{mM}$ EGTA, and $0.1 \mathrm{mM}$ DTT. Representative curves show the increase in fluorescence intensity upon addition of either wt troponin (circles) or mutant troponin (squares).

were similar to those obtained in parallel titrations (not shown) with bovine troponin that was purified as a complex from bovine hearts: $1.8 \pm 0.4 \times 10^{6} \mathrm{M}^{-1}$ in the presence of EGTA and $2.0 \pm 0.2 \times 10^{6} \mathrm{M}^{-1}$ in the presence of $\mathrm{Ca}^{2+}$.

Effect of TnT I91N mutation on binding of tropomyosin to actin. Actin binding of wt and mutant troponin-tropomyosin complexes is shown in the representative experiment in Fig. 3. It has been established that troponin increases the affinity of tropomyosin for actin by 100-500-fold and this effect is largely attributable to $\operatorname{TnT}(12,14,24)$. To determine whether the I91N mutation altered this function of TnT, troponin-induced binding of tropomyosin to actin was investigated. Under the conditions of this experiment $(0.3 \mathrm{M} \mathrm{KCl})$ tropomyosin-actin

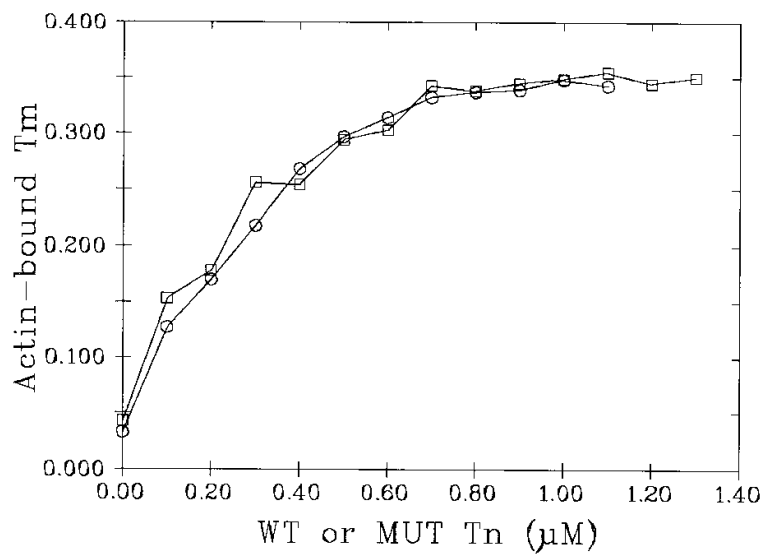

Figure 3. Induction of $\left[{ }^{3} \mathrm{H}\right]$ tropomyosin binding to actin by wt or mutant troponin. Troponin-induced tropomyosin binding to actin was monitored as the concentration of troponin was increased. Wt troponin (circles) and mutant troponin (squares). Conditions: $10 \mathrm{mM}$ Tris$\mathrm{HCl}(\mathrm{pH} 7.5), 3 \mathrm{mM} \mathrm{MgCl}_{2}, 300 \mathrm{mM} \mathrm{KCl}, 0.5 \mathrm{mM}$ EGTA, $5 \mu \mathrm{M} \mathrm{F}$-actin, $0.6 \mu \mathrm{M}\left[{ }^{3} \mathrm{H}\right]$ tropomyosin, and $25^{\circ} \mathrm{C}$. The wt and mutant troponin curves were indistinguishable.

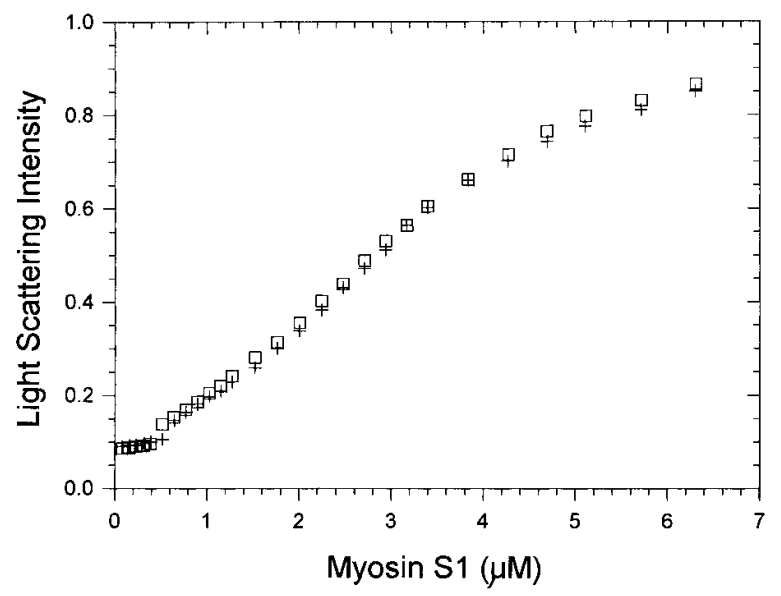

Figure 4. Binding of rabbit skeletal myosin S-1 to the actin-tropomyosin-troponin complex. Myosin S-1 binding to the thin filament complex was measured by $90^{\circ}$ light-scattering intensity at $400 \mathrm{~nm}$. In the absence of the thin filament, the myosin S-1 has negligible light scattering under these conditions. The points show similar cooperative transitions for myosin S-1 binding to thin filaments containing wt troponin (squares) or mutant troponin (crosses). Conditions were as described in Methods.

binding is weak in the absence of troponin, which makes it easy to study the troponin-induced increase in tropomyosinactin affinity. At $25^{\circ} \mathrm{C}$ with EGTA, the I91N mutation did not alter troponin's induction of $\left[{ }^{3} \mathrm{H}\right]$ tropomyosin binding to actin. For both wt and mutant reconstituted troponin, tropomyosinactin binding reached the same level and required the same troponin concentration. At a more physiological temperature, $37^{\circ} \mathrm{C}$, very similar results were found (data not shown). Despite TnT's primary role in mediating troponin binding to tropomyosin and in the induction of troponin-tropomyosin complex binding to actin, this TnT mutation had no effect on these processes in vitro. Similar results were obtained in presence of $\mathrm{Ca}^{2+}$ (data not shown).

Effect of TnT mutation on rabbit skeletal muscle myosinsubfragment 1 binding to actin-tropomyosin-troponin. Light scattering can be used to detect the binding of myosin S-1 to the actin-tropomyosin-troponin complex (33). Addition of myosin S-1 to the thin filament results in very cooperative binding (34-36). Moreover, this cooperativity is believed to be partially mediated by direct contacts between adjacent troponin-tropomyosin complexes on the thin filament $(37,38)$. These contacts involve the amino terminal region of TnT (27), which includes the site of the Ile to Asn mutation (see Discussion). Therefore, the effect of this mutation on cooperative binding of myosin S-1 to the thin filament was evaluated by light scattering. (Cardiac myosin S-1 has high turbidity in the absence of actin under these conditions, so skeletal muscle myosin S-1 was used instead.) A cooperative transition was observed with increasing myosin S-1 concentrations (Fig. 4), demonstrated by the initial lag in the binding curves. The light scattering profiles of reconstituted wt and mutant troponins were indistinguishable under the conditions studied $(150 \mathrm{mM}$ $\mathrm{NaCl}, 2 \mathrm{mM}$ ADP, and 0.1 mM EGTA). Similar to Figs. 2 and 3 , where the mutation demonstrated no effects on troponintropomyosin binding or on troponin-induced tropomyosin binding to actin, the myosin S-1-induced change in the state of tropomyosin-troponin on actin also showed no significant dif- 


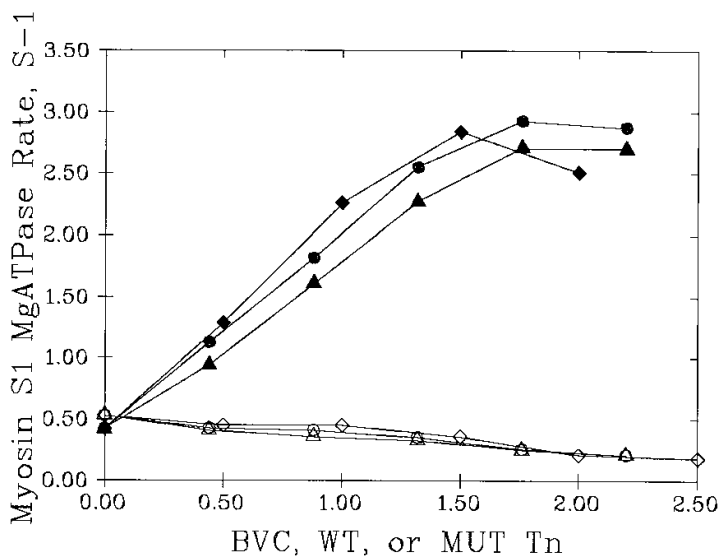

Figure 5. Effect of TnT I91N mutation on $\mathrm{Ca}^{2+}$ sensitive regulation of the myosin S-1 thin filament MgATPase rate. The figure shows representative myosin S-1-thin filament MgATPase data in the presence of bovine cardiac troponin, wt reconstituted troponin, or I91N $\mathrm{TnT}$ reconstituted troponin. Closed symbols, $0.1 \mathrm{mM} \mathrm{CaCl}_{2}$, showing troponin's activating effect; open symbols, 0.5 mM EGTA, showing troponin's inhibitory effect; diamonds, bovine cardiac whole troponin; circles, reconstituted troponin with I91N rat cardiac TnT; triangles, reconstituted troponin with wt TnT. The mutant troponin activates and inhibits the myosin S-1 MgATPase rate as well as wt or bovine cardiac troponin.

ference between the wt and mutant troponins. The results in Fig. 4 suggest that the mutation does not alter crossbridgeinduced thin filament activation, believed to be an important aspect of muscle regulation $(36,39,40)$.

$191 N$ TnT mutation has no effect on MgATPase rate activation. To further study the effect of $\mathrm{I} 91 \mathrm{~N}$ mutation on protein function, troponin's ability to regulate the myosin S-1 ATPase rate was studied. The primary function of troponin is to confer $\mathrm{Ca}^{2+}$ sensitivity to the force-producing interactions of actin and myosin (41). Troponin also confers $\mathrm{Ca}^{2+}$ sensitivity to an in vitro correlate of muscle contraction, the MgATPase rate of actin-tropomyosin-myosin S-1. As shown in Fig. 5, the mutation didn't alter MgATPase rate activation by troponin in the presence of $\mathrm{Ca}^{2+}$, nor the concentration of troponin needed to achieve it. Similarly, the mutation didn't alter troponin's ability to inhibit the $\mathrm{MgATPase}$ rate in the absence of $\mathrm{Ca}^{2+}$.

Comparison of myosin S-1 MgATPase activation by $\mathrm{Ca}^{2+}$ between wt and mutant troponin. A more subtle effect of a troponin mutation might be to influence thin filament activation of the myosin S-1 MgATPase rate in the presence of intermediate $\mathrm{Ca}^{2+}$ concentrations; an effect on either the apparent $\mathrm{Ca}^{2+}$ affinity or the cooperativity of activation. Alterations in the hypervariable region of TnT, located immediately $\mathrm{NH}_{2}-$ terminal to the site of the Ile to Asn mutation (see Fig. 8), can have subtle effects on the $\mathrm{Ca}^{2+}$-dependence of MgATPase activation $(18,42,43)$ or of muscle tension (44-48). Thin filaments were prepared containing actin, tropomyosin, and ternary troponin complexes with either wt or mutant TnT. Fig. 6 shows that $\mathrm{Ca}^{2+}$ cooperatively activated the thin filamentmyosin S-1 MgATPase rate. The curves shown for thin filaments containing wt and mutant TnTs are indistinguishable. The $\mathrm{K}_{\mathrm{app}}$ for activation (apparent $\mathrm{Ca}^{2+}$ affinity), the cooperativity of activation (the transition steepness), and the fully activated enzymatic rate are all unchanged by the presence of the I91N mutation.

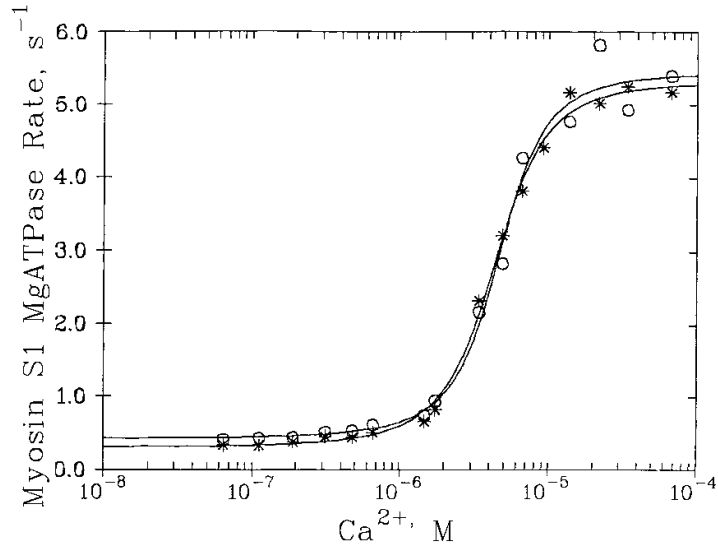

Figure 6. Comparison of myosin S-1 MgATPase activation between wt and mutant troponin as a function of the $\mathrm{Ca}^{2+}$ concentration. $\mathrm{Ca}^{2+}$-sensitive regulation of the myosin S-1 MgATPase rate by wt (circles) and $\mathrm{I} 91 \mathrm{~N} \mathrm{TnT} \mathrm{(asterisks)} \mathrm{reconstituted} \mathrm{troponins} \mathrm{at} 25^{\circ} \mathrm{C}$ in the presence of $20 \mathrm{mM}$ Imidazole ( $\mathrm{pH} 7.5), 6.5 \mathrm{mM} \mathrm{KCl}, 3.5 \mathrm{mM}$ $\mathrm{MgCl}_{2}, 0.5 \mathrm{mM}$ dibromo-BAPTA, $0.3 \mu \mathrm{M}$ myosin S-1, $2 \mu \mathrm{M}$ bovine cardiac tropomyosin, $2 \mu \mathrm{M}$ mutant or wt troponin, and $14 \mu \mathrm{M}$ rabbit skeletal F-actin. The mutation had no observable effect on the $K_{\text {app }}$ of activation, the cooperativity of activation, or the maximum enzymatic rate. Data is fit to Eq. 12 of Tobacman and Sawyer (23) with an apparent $\mathrm{Ca}^{2+}$ affinity of $0.23 \times 10^{6} \mathrm{M}^{-1}$ and a cooperativity parameter of 5.7 .

Thin filaments containing the mutant TnT move faster in an in vitro motility assay. Despite the normal behavior of the mutant TnT in all the experiments described above, it was possible to distinguish the wt and mutant proteins in one respect. The in vivo regulation of muscle contraction can be investigated in vitro not only in an ATPase assay, but also in the regulation of movement as assessed by video epifluorescence mi-

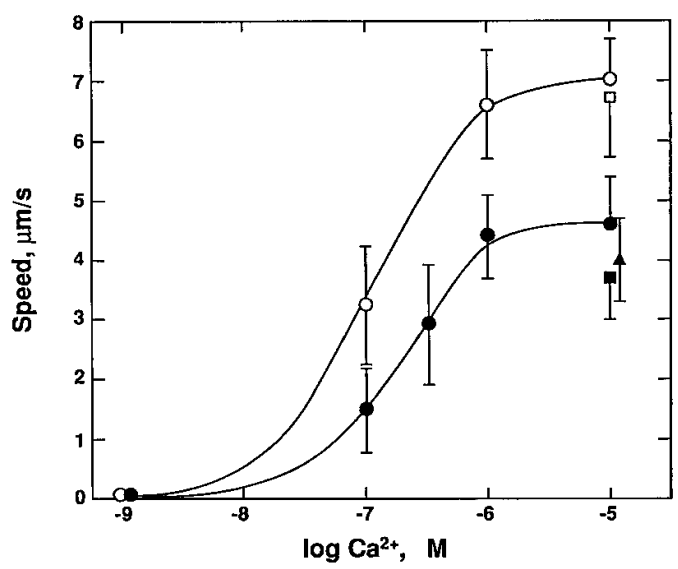

Figure 7. Effect of the TnT mutation on in vitro motility. Movement of fluorescently labeled thin filaments was caused by interaction with heavy meromyosin that was bound to a microscope coverslip. Thin filament speed is plotted as a function of the free $\mathrm{Ca}^{2+}$ concentration. Mean velocities \pm standard deviations are shown. Since each mean represents $>100$ filaments, the standard errors are much smaller than the brackets shown. Circles $v s$ squares represent different pairs of protein preparations; open symbols, thin filaments reconstituted from actin, tropomyosin, and troponin containing I91N mutant TnT; closed symbols, identical thin filaments except the troponin-contained wt TnT; triangle, identical thin filaments except that troponin was whole bovine cardiac troponin. Solid lines are free hand curves. 
croscopy $(26,49,50)$. The results of such an analysis are shown in Fig. 7. Neither thin filaments with wt nor with mutant TnT move in the presence of very low $\mathrm{Ca}^{2+}$ concentration, and both types of thin filaments move faster over the heavy meromyosin-coated surface as the $\mathrm{Ca}^{2+}$ concentration is increased. In agreement with the ATPase results (Fig. 6), the $\mathrm{Ca}^{2+} \mathrm{K}_{\text {app }}$ is not affected by the mutation. However, the presence of the I91N mutation in TnT (open symbols) results in 50\% faster speeds than found when the mutation is absent (filled symbols). This difference was not only significant statistically (the standard error of the mean is small and $t$ test comparisons at pCa 7, 6, and 5 show effects of the mutation with $P<0.01$ ), it was also reproducible. Similar results were obtained with a separately prepared set of wt and mutant troponins (squares). Also, the speed of filaments containing wt TnT was similar to that found for thin filaments containing bovine whole troponin (triangles).

\section{Discussion}

TnT is one of three subunits of troponin, which regulates cardiac and skeletal muscle contraction via reversible binding of $\mathrm{Ca}^{2+}$ to another troponin subunit, $\operatorname{TnC}(9,11,51-53)$. TnT is highly elongated and appears rodlike in electron microscopic (54) and low resolution crystallographic studies (30). Although TnT may play a role in transmitting the regulatory $\mathrm{Ca}^{2+}$ signal $(24,55)$, its most clearly demonstrated function is to promote assembly of the regulatory troponin-tropomyosin apparatus onto the actin filament (reviewed in 11). TnT interacts directly with all four of the other thin filament proteins: tropomyosin, actin, TnC, and TnI.

The relationship between the structure and the function of troponin subunits has been most thoroughly explored using rabbit fast skeletal muscle troponin. In Fig. 8, biologically significant mutations in various isoforms of TnT are mapped to the amino acid sequence and functional regions of rabbit skeletal muscle TnT. The most $\mathrm{NH}_{2}$-terminal of the HCM-causing mutations (arrows) is the one studied in this report. Biochemical evidence suggests that this mutation (corresponding to site I49N in rabbit) would cause only subtle changes in function. Deletion of rabbit residues 1-69 has at most a twofold effect on troponin-tropomyosin binding (14), troponin-induced tropomyosin-actin binding (12-14), and the cooperativity of thin filament assembly $(12,13)$. Fujita et al. (15), reported normal function for a truncated form of rabbit skeletal muscle TnT that was missing residues $1-58$, and includes the amino acid corresponding to Ile79 in human cardiac TnT. These previous studies are consistent with the present results, in which the Ile to Asn mutation at this site has no effect on troponin-tropomyosin binding, troponin-induced tropomyosin-actin binding, $\mathrm{Ca}^{2+}$ activation of the myosin-thin filament ATPase rate, the degree of cooperativity and apparent $\mathrm{Ca}^{2+}$ affinity for this activation, or cooperative myosin S-1 binding to the thin filament.

On the other hand, one defect in function was found (Fig. 7) and this effect may provide a pathophysiological explanation for HCM in the individuals with this mutation. It is notable that the HCM patients having TnT mutations exhibit less cardiac hypertrophy than most other patients with HCM (2). Enhanced motility, as opposed to the inhibited motility generally seen with myosin heavy chain gene mutations $(5,6,8)$ could cause a hypercontractile state directly, instead of causing it indirectly via compensatory hypertrophy. It remains to be

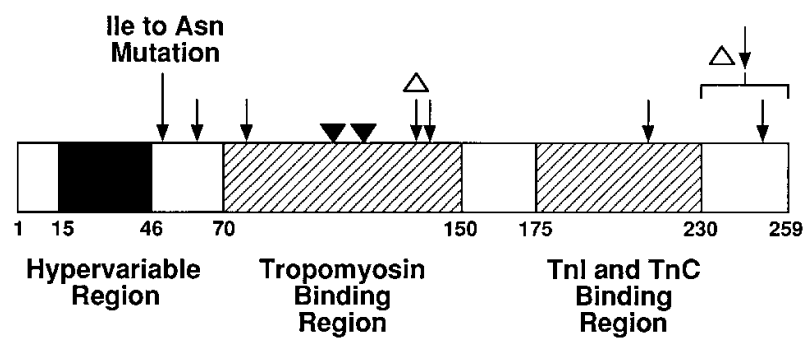

Figure 8. Mapping of human and Drosophila TnT mutations to functional regions in rabbit fast skeletal muscle TnT. Arrows indicate the positions in rabbit fast skeletal muscle TnT (76) that correspond to human cardiac TnT (57) mutations linked to familial hypertrophic cardiomyopathy $(2,3)$. The longer arrow is the mutation studied in this report. The filled triangles $(\boldsymbol{\nabla})$ indicate the positions of D. melanogaster point mutations that cause flight muscle degeneration upon onset of contraction $(73,77)$. Deleted residues or sequences are indicated by the open triangles $(\Delta)$. The tertiary structure of troponin is unknown, so the proposed regions for interaction of TnT with other proteins are approximate (reviewed in reference 11). Also, tropomyosin binds to many regions of TnT; only the tightest-binding region is indicated. Human cardiac TnT and rabbit fast skeletal muscle TnT are significantly homologous throughout their sequences, except in the indicated region that is hypervariable among TnTs in general.

established, however, whether the other HCM-causing TnT mutations produce accelerated in vitro motility, and mechanical studies of hearts containing TnT mutations are not yet reported.

Despite the great efforts that have been devoted to understanding thin filament-based regulation (see reviews 9-11, 52, 53), the increased sliding speed seen in Fig. 7 cannot easily be related to previous work. The in vitro motility speed is analogous to the unloaded shortening velocity of a muscle fiber (56), although this analogy is imperfect (25). Faster movement in the presence of saturating $\mathrm{Ca}^{2+}$ suggests an increase in either the distance per cross-bridge stroke (unlikely for a TnT point mutation), or the cross-bridge release rate at the end of the power stroke. It would be unexpected but important if TnT modulates this kinetic step, because it would provide the possibility for physiological regulation of the unloaded shortening velocity. In this regard, the mutation site is very close to the hypervariable region of TnT that is developmentally regulated by alternative splicing mechanisms $(27,57-59)$. Indeed, subtle changes in actin-myosin interactions $(18,42)$ and the $\mathrm{Ca}^{2+}$ sensitivity of muscle tension $(44,46,47)$ are produced by amino acid sequence variations in this region, and possibly also by phosphorylation (60).

It is notable that the altered speed produced by the TnT mutation studied in this report has no correlation with any of the other biochemical experiments performed. This precludes detailed mechanistic conclusions about the enhanced motility, but is not contradictory because the rate-limiting step for the actin-myosin MgATPase reaction is not the same as the kinetic step limiting the unloaded shortening velocity (61). In fact, there is ample precedent for discrepancy between effects on in vitro motility and effects on actin-activated $\mathrm{MgATPase}$ rates. For example, increasing the charge of the amino-terminal region of yeast actin had little effect on motility, but a very large effect on the actin-activated MgATPase rate (62). Similar effects were found for phosphorylation of smooth muscle myosin light chain 2 on threonine 18 (63). Conversely, large effects on motility and small effects on actin-activated MgATPase 
rate were found for several other alterations of myosin: truncation of the motor domain (64), removal of light chains (65), myosin isoform comparisons (66), and phosphorylation of monomeric Acanthamoeba myosin II (67). Also, ionic strength variation has a greater effect on motility than would be expected from its effect on actin-activated MgATPase rates (25). Finally, the unloaded shortening velocity of different muscle fibers does not scale to the actin-activated MgATPase rate, which is much too slow, but rather to the rate of ADP release from actomyosin (68). Similarly, the in vitro motility speed is limited by a kinetic step that is very fast $(4,000 \mathrm{~nm} / \mathrm{s}$ motility speed/10-nm crossbridge stroke translocation distance = $250 \mathrm{~s}^{-1}$ ), much faster than the actin-activated MgATPase rate.

The TnT mutation in the present study has no effect on the interactions of troponin with tropomyosin and actin, nor on the interactions of rigor crossbridges with the thin filament, nor on the apparent $\mathrm{Ca}^{2+}$ affinity of TnC. This suggests the mutation has little effect on the overall quaternary structure of the thin filament, and raises the interesting possibility that the enhanced motility is due to a local effect on actin-myosin kinetics. In other words, the accelerated in vitro motility could be caused by a local acceleration affecting one or two of the seven actins in a regulatory unit, rather than alteration of actin-myosin kinetics for the entire thin filament. To evaluate this hypothesis, however, further study is needed.

Three of the mutations in man and two mutations in Drosophila melanogaster (filled diamonds in Fig. 8) are found in a region crucial for TnT function, spanning amino acids 70150 in the rabbit isoform. This is the region of troponin that binds most tightly to tropomyosin $(14,24,30,31,69,70)$ and that is most important for promoting tropomyosin-actin binding (12-14), although the COOH-terminal region of TnT also binds to tropomyosin $(14,30-32,69-71)$. Homozygous missense mutations in this region of D. melanogaster TnT (Fig. 8) lead to rapid muscle degeneration upon onset of flight muscle contraction. The more $\mathrm{COOH}$-terminal TnT mutations found in HCM are either within the TnT region most clearly shown to interact with the other troponin subunits (residues 176-230, as reviewed in (11), or else alter the adjacent sequence (residues 230 259), in which isoforms are known to affect $\mathrm{Ca}^{2+}$ affinity (72).

In D. melanogaster, flight muscle ultrastructure and function are profoundly impaired when one copy of the actin, myosin, tropomyosin, or TnT genes is disrupted (73-75), implying that expression levels for each of these proteins is critical. These data are consistent with the absence of known TnT or tropomyosin knock-out mutations in HCM kindreds, which would be fatal in heterozygotes if the insect results are predictive of the effects in man. Rather, HCM appears to result from more moderate defects in protein function, such as found in the current study.

Finally, the relatively subtle effects of the TnT I91N mutation require restatement of the evidence for a causative connection between TnT mutations and hypertrophic cardiomyopathy. The crucial point is that genetic linkage has been established between the disease and several different TnT mutations in several different kindreds (2). The genetic linkage studies for any single TnT mutation might not reflect causation, but merely proximity to the true disease-causing locus. However, the combined results in the different kindreds provide a convincing connection between HCM and mutations in the cardiac TnT gene. Our finding that one of these mutations results in faster in vitro motility is further proof of disease cau- sation. Furthermore, the data suggest that HCM can be caused not only by slowed actin-myosin kinetics, as shown previously for myosin mutations, but alternatively by a kinetic enhancement of actin-myosin cycling, producing accelerated motion and altered cardiac contraction.

\section{Acknowledgments}

We thank Dr. Jim Jung-Ching Lin of the University of Iowa for his generous gift of rat cardiac TnT cDNA (30).

This work was supported by National Institutes of Health grants HL-38834 (L. Tobacman) and AR-30988 (E. Homsher).

\section{References}

1. Geisterfer-Lowrance, A.A., S. Kass, G. Tanigawa, H.P. Vosberg, W. McKenna, C.E. Seidman, and J.G. Seidman. 1990. A molecular basis for familial hypertrophic cardiomyopathy: a beta cardiac myosin heavy chain gene missense mutation. Cell. 62:999-1006.

2. Watkins, H., W.J. McKenna, L. Thierfelder, H.J. Suk, R. Anan, A O'Donoghue, P. Spirito, A. Matsumori, C.S. Moravec, J.G. Seidman, et al. 1995. Mutations in genes for cardiac troponin $\mathrm{T}$ and a-tropomyosin in hypertrophic cardiomyopathy. N. Engl. J. Med. 332:1058-1064.

3. Thierfelder, L., H. Watkins, C. MacRae, R. Lamas, W. McKenna, H.-P. Vosberg, J.G. Seidman, and C.E. Seidman. 1994. $\alpha$-Tropomyosin and cardiac troponin $\mathrm{T}$ mutations cause familial hypertrophic cardiomyopathy: a disease of the sarcomere. Cell. 77:701-712.

4. Watkins, H., D. Conner, L. Thierfelder, J.A. Jarcho, C. MacRae, W.J. McKenna, B.J. Maron, J.G. Seidman, and C.E. Seidman. 1995. Mutations in the cardiac myosin binding protein-C gene on chromosome 11 cause familial hypertrophic cardiomyopathy. Nat. Genet. 11:434-437.

5. Sweeney, H.L., A.J. Straceski, L.A. Leinwand, B.A. Tikunov, and L. Faust. 1994. Heterologous expression of a cardiomyopathic myosin that is defective in its actin interaction. J. Biol. Chem. 269:1603-1605.

6. Cuda, G., L. Fananapazir, W.S. Zhu, J.R. Sellers, and N.D. Epstein. 1993. Skeletal muscle expression and abnormal function of $\beta$-myosin in hypertrophic cardiomyopathy. J. Clin. Invest. 91:2861-2865.

7. Rayment, I., H.M. Holden, J.R. Sellers, L. Fanapazir, and N.D. Epstein. 1995. Structural interpretation of the mutations in the b-cardiac myosin that have been implicated in familial hypertrophic cardiomyopathy. Proc. Natl. Acad. Sci. USA. 92:3864-3868.

8. Lankford, E.B., N.D. Epstein, L. Fananapazir, and H.L. Sweeney. 1995. Abnormal contractile properties of muscle fibers expressing $\beta$-myosin heavy chain gene mutations in patients with hypertrophic cardiomyopathy. J. Clin. Invest. 95:1409-1414.

9. Zot, A.S., and J.D. Potter. 1987. Structural aspects of troponin-tropomyosin regulation of skeletal muscle contraction. Annu. Rev. Biophys. Biophys. Chem. 16:535-559.

10. Chalovich, J.M. 1992. Actin mediated regulation of muscle contraction. Pharmacol. \& Ther. 55:95-148.

11. Tobacman, L.S. 1996. Thin filament mediated regulation of cardiac contraction. Annu. Rev. Physiol. 58:447-481.

12. Hill, L.E., J.P. Mehegan, C.A. Butters, and L.S. Tobacman. 1992. Analysis of troponin-tropomyosin binding to actin. Troponin does not promote interactions between tropomyosin molecules. J. Biol. Chem. 267:16106-16113.

13. Willadsen, K.A., C.A. Butters, L.E. Hill, and L.S. Tobacman. 1992. Effects of the amino-terminal regions of tropomyosin and troponin $\mathrm{T}$ on thin filament assembly. J. Biol. Chem. 267:23746-23752.

14. Fisher, D., G. Wang, and L.S. Tobacman. 1995. N-terminal truncation of skeletal muscle troponin $\mathrm{T}$ does not alter the $\mathrm{Ca}^{2+}$ sensitivity of thin filament assembly. J. Biol. Chem. 270:25455-25460.

15. Fujita, S., K. Maeda, and Y. Maeda. 1992. Expression in Escherichia coli and a functional study of a b-troponin T $25 \mathrm{kDa}$ fragment of rabbit skeletal muscle. J. Biochem. (Tokyo). 112:306-308.

16. Studier, F.W., A.H. Rosenberg, J.J. Dunn, and J.W. Dubendorff. 1990. Use of T7 RNA polymerase to direct expression of cloned genes. Methods Enzymol. 185:60-89.

17. Sanger, F., S. Nikben, and A.R. Coulsen. 1977. DNA sequencing with chain-terminating inhibitors. Proc. Natl. Acad. Sci. USA. 74:5463-5467.

18. Tobacman, L.S., and R. Lee. 1987. Isolation and functional comparison of bovine cardiac troponin T isoforms. J. Biol. Chem. 262:4059-4064.

19. Weeds, A.G., and R.S. Taylor. 1975. Separation of subfragment-1 isoenzymes from rabbit skeletal muscle myosin. Nature (Lond.). 257:54-56.

20. Spudich, J.A., and S. Watt. 1971. The regulation of rabbit skeletal muscle contraction. I. Biochemical studies of the interaction of the tropomyosintroponin complex with actin and the proteolytic fragments of myosin. J. Biol. Chem. 246:4866-4871. 
21. Pollard, T.D., and E.D. Korn. 1973. Acanthamoeba myosin. Isolation from Acanthamoeba castellani of an enzyme similar to muscle myosin. J. Biol. Chem. 248:4682-4690.

22. Tobacman, L.S. 1987. Activation of actin-cardiac myosin subfragment 1 MgATPase rate by $\mathrm{Ca}^{2+}$ shows cooperativity intrinsic to the thin filament. Biochemistry. 26:492-497.

23. Tobacman, L.S., and D. Sawyer. 1990. Calcium binds cooperatively to the regulatory sites of the cardiac thin filament. J. Biol. Chem. 265:931-939.

24. Dahiya, R., C.A. Butters, and L.S. Tobacman. 1994. Equilibrium linkage analysis of cardiac thin filament assembly. Implications for the regulation of muscle contraction. J. Biol. Chem. 269:29457-29461.

25. Homsher, E., F. Wang, and J.R. Sellers. 1992. Factors affecting movement of F-actin filaments propelled by skeletal muscle heavy meromyosin. Am. J. Physiol. 262:C714-C723.

26. Homsher, E., B. Kim, A. Bobkova, and L.S. Tobacman. 1996. Calcium regulation of thin filament movement in an in vitro motility assay. Biophys. $J$. In press.

27. Jin, J.-P., and J.J.-C. Lin. 1989. Isolation and characterization of cDNA clones encoding embryonic and adult isoforms of rat cardiac troponin T. J. Biol. Chem. 264:14471-14477.

28. Jin, J.-P., Q.-Q. Huang, H.-I. Yeh, and J.J.-C. Lin. 1992. Complete nucleotide sequence and structural organization of rat cardiac troponin T gene. A single gene generates embryonic and adult isoforms via developmentally regulated alternative splicing. J. Mol. Biol. 227:1269-1276.

29. Leszyk, J., R. Dumaswala, J.D. Potter, N.B. Gusev, A.D. Verin, L.S. Tobacman, and J.H. Collins. 1987. Bovine cardiac troponin T: amino acid sequences of the two isoforms. Biochemistry. 26:7035-7042.

30. White, S.P., C. Cohen, and J. Phillips. 1987. Structure of co-crystals of tropomyosin and troponin. Nature (Lond.). 325:826-828.

31. Ishii, Y., and S.S. Lehrer. 1991. Two-site attachment of troponin to pyrene-labeled tropomyosin. J. Biol. Chem. 266:6894-6903.

32. Pearlstone, J.R. and L.B. Smillie. 1982. Binding of troponin-T fragments to several types of tropomyosin. Sensitivity to $\mathrm{Ca}^{2+}$ in the presence of troponinC. J. Biol. Chem. 257:10587-10592.

33. Stein, L.A., R.P.J. Schwarz, P.B. Chock, and E. Eisenberg. 1979. Mechanism of actomyosin adenosine triphosphatase. Evidence that adenosine 5'triphosphate hydrolysis can occur without dissociation of the actomyosin complex. Biochemistry. 18:3895-3909.

34. Greene, L.E., and E. Eisenberg. 1980. Cooperative binding of myosin subfragment-1 to the actin-troponin-tropomyosin complex. Proc. Natl. Acad. Sci. USA. 77:2616-2620.

35. Trybus, K.M. and E.W. Taylor. 1980. Kinetic studies of the cooperative binding of subfragment 1 to regulated actin. Proc. Natl. Acad. Sci. USA. 77: 7209-7213.

36. McKillop, D.F.A., and M.A. Geeves. 1993. Regulation of the interaction between actin and myosin subfragment 1 : evidence for three states of the thin filament. Biophys. J. 65:693-701.

37. Hill, T.L., E. Eisenberg, and L.E. Greene. 1980. Theoretical model for cooperative equilibrium binding of myosin subfragment- 1 to the actin-troponin-tropomyosin complex. Proc. Natl. Acad. Sci. USA. 77:3186-3190.

38. Pan, B.-S., A.M. Gordon, and Z. Luo. 1989. Removal of tropomyosin overlap modifies cooperative binding of myosin S-1 to reconstituted thin filaments of rabbit striated muscle. J. Biol. Chem. 264:8495-8498.

39. Lehrer, S.S. 1994. The regulatory switch of the muscle thin filament: $\mathrm{Ca}^{2+}$ or myosin heads? J. Muscle Res. Cell Motil. 15:232-236.

40. Williams, D.L., L.E. Greene, and E. Eisenberg. 1988. Cooperative turning on of myosin subfragment 1 adenosine triphosphatase activity by the troponin-tropomyosin-actin complex. Biochemistry. 27:6897-6993.

41. Ebashi, S., M. Endo, and I. Ohtsuki. 1969. Control of muscle contraction. O. Rev. Biophys. 2:351-384.

42. Tobacman, L.S. 1988. Structure-function studies of the amino-terminal region of bovine cardiac troponin T. J. Biol. Chem. 263:2668-2672.

43. Pan, B.-S., A.M. Gordon, and J.D. Potter. 1991. Deletion of the first 45 $\mathrm{NH}_{2}$-terminal residues of rabbit skeletal muscle troponin $\mathrm{T}$ strengthens binding of troponin to immobilized tropomyosin. J. Biol. Chem. 266:12432-12438.

44. Reiser, P.J., M.L. Greaser, and R.L. Moss. 1992. Developmental changes in troponin $\mathrm{T}$ isoform expression and tension production in chicken single skeletal muscle fibers. J. Physiol. (Lond.). 449:573-588.

45. McAuliffe, J.J., L.Z. Gao, and R.J. Solaro. 1990. Changes in myofibrillar activation and troponin $\mathrm{C} \mathrm{Ca}^{2+}$ binding associated with troponin $\mathrm{T}$ switching in developing rabbit heart. Circ. Res. 66:1204-1216.

46. Nassar, R., N.N. Malouf, M.B. Kelly, A.E. Oakeley, and P.A. Anderson. 1991. Force-pCa relation and troponin T isoforms of rabbit myocardium. Circ. Res. 69:1470-1475.

47. Schachat, F.H., M.S. Diamond, and P.W. Brandt. 1987. Effect of different troponin T-tropomyosin combinations on thin filament activation. J. Mol. Biol. 198:551-554.

48. Grieg, A., Y. Hirschberg, P.A.W. Anderson, C. Hainsworth, N.N. Malouf, A.E. Oakeley, and B.K. Kay. 1994. Molecular basis of cardiac troponin T isoform heterogeneity in rabbit heart. Circ. Res. 74:41-47.

49. Honda, H., and S. Asakura. 1989. Calcium triggered movement of regulated actin in vitro. J. Mol. Biol. 205:677-683.
50. Fraser, I.D.C., and S.B. Marston. 1995. In vitro motility analysis of actintropomyosin regulation by troponin and calcium. J. Biol. Chem. 270:7836-7841.

51. Putkey, J.A., H.L. Sweeney, and S.T. Campbell. 1989. Site-directed mutation of the trigger calcium-binding sites in cardiac troponin C. J. Biol. Chem. 264:12370-12378.

52. Leavis, P.C., and J. Gergely. 1984. Thin filament proteins and thin filament-linked regulation of vertebrate muscle contraction. CRC Crit. Rev. Biochem. 16:235-305.

53. Ashley, C.C., I.P. Mulligan, and T.J. Lea. 1991. $\mathrm{Ca}^{2+}$ and activation mechanisms in skeletal muscle. Q. Rev. Biophys. 24:1-73.

54. Flicker, P.F., G.N. Phillips, Jr., and C. Cohen. 1982. Troponin and its interactions with tropomyosin: an electron microscope study. J. Mol. Biol 162:495-501.

55. Potter, J.D., Z. Sheng, B.-S. Pan, and J. Zhao. 1995. A direct regulatory role for troponin $\mathrm{T}$ and a dual role for troponin $\mathrm{C}$ in the $\mathrm{Ca}^{2+}$ regulation of muscle contraction. J. Biol. Chem. 270:2557-2562.

56. Sheetz, M.P., and J.A. Spudich. 1983. Movement of myosin-coated fluorescent beads on actin cables in vitro. Nature (Lond.). 303:31-35.

57. Mesnard, L., F. Samson, I. Espinasse, J. Durand, J.-Y. Neveux, and J.-J. Mercadier. 1993. Molecular cloning and developmental expression of human cardiac troponin T. FEBS Lett. 328:139-144.

58. Cooper, T.A., and C.P. Ordahl. 1985. A single cardiac troponin T gene generates embryonic and adult isoforms via developmentally regulated alternate splicing. J. Biol. Chem. 260:11140-11148.

59. Breitbart, R.E., A. Andreadis, and B. Nadal-Ginard. 1987. Alternative splicing: a ubiquitous mechanism for the generation of multiple protein isoforms from single genes. Annu. Rev. Biochem. 56:467-495.

60. Noland, T.A., Jr., and J.F. Kuo. 1992. Protein kinase C phosphorylation of cardiac troponin $\mathrm{T}$ decreases $\mathrm{Ca}\left({ }^{2+}\right)$-dependent actomyosin $\mathrm{MgATPase}$ activity and troponin T binding to tropomyosin-F-actin complex. Biochem. J. 288: 123-129.

61. Adelstein, R.S., and E. Eisenberg. 1980. Regulation and kinetics of the actin-myosin-ATP interaction. Annu. Rev. of Biochem. 49:921-956.

62. Cook, R.K., D. Root, C. Miller, E. Reisler, and P.A. Rubenstein. 1993. Enhanced stimulation of myosin subfragment 1 ATPase activity by addition of negatively charged residues to the yeast actin $\mathrm{NH}_{2}$ terminus. J. Biol. Chem. 268: $2410-2415$.

63. Umemoto, S., A.R. Bengur, and J.R. Sellers. 1989. Effect of multiple phosphorylations of smooth muscle and cytoplasmic myosins on movement in an in vitro motility assay. J. Biol. Chem. 264:1431-1436.

64. Waller, G.S., G. Ouyang, J. Swafford, P. Vibert, and S. Lowey. 1995. A minimal motor domain from chicken skeletal muscle myosin. J. Biol. Chem. 270:15348-15352.

65. Lowey, S., G.S. Waller, and K.M. Trybus. 1993. Skeletal muscle myosin light chains are essential for physiological speeds of shortening. Nature (Lond.). 365:454-456.

66. Lowey, S., G.S. Waller, and K.M. Trybus. 1993. Function of skeletal muscle myosin heavy chain and light chain isoforms by an in vitro motility assay. J. Biol. Chem. 268:20414-20418.

67. Ganguly, C., I.C. Baines, E.D. Korn, and J.R. Sellers. 1992. Regulation of the actin-activated ATPase and in vitro motility activities of monomeric and filamentous Acanthamoeba myosin. J. Biol. Chem. 267:20900-20904.

68. Siemankowski, R.F., M.O. Wiseman, and H.D. White. 1985. ADP dissociation from actomyosin subfragment 1 is sufficiently slow to limit the unloaded shortening velocity in vertebrate muscle. Proc. Natl. Acad. Sci. USA. 82:658662

69. Morris, E.P., and S.S. Lehrer. 1984. Troponin-tropomyosin interactions. Fluorescence studies of the binding of troponin, troponin $\mathrm{T}$, and chymotryptic troponin $\mathrm{T}$ fragments to specifically labeled tropomyosin. Biochemistry. 23: 2214-2220.

70. Mak, A.S., and L.B. Smillie. 1981. Structural interpretation of the twosite binding of troponin on the muscle thin filament. J. Mol. Biol. 149:541-550.

71. Tanokura, M., Y. Tawada, A. Ono, and I. Ohtsuki. 1983. Chymotryptic subfragments of troponin $\mathrm{T}$ from rabbit skeletal muscle. Interactions with tropomyosin, troponin I and troponin C. J. Biochem. (Tokyo). 93:331-337.

72. Pan, B.-S., and J.D. Potter. 1992. Two genetically expressed troponin T fragments representing $\alpha$ and $\beta$ isoforms exhibit functional differences. J. Biol. Chem. 267:23052-23056.

73. Fyrberg, E., C.C. Fyrberg, C. Beall, and D.L. Saville. 1990. Drosophila melanogaster troponin-T mutations engender three distinct syndromes of myofibrillar abnormalities. J. Mol. Biol. 216:657-675.

74. Beall, C.J., M.A. Sepanski, and E.A. Fyrberg. 1989. Genetic dissection of Drosophila myofibril formation: effects of actin and myosin heavy chain null alleles. Genes \& Devel. 3:131-140.

75. Molloy, J., A. Kreuz, R. Miller, T. Tansey, and D. Maughan. 1993. Effects of tropomyosin deficiency in flight muscle of Drosophila melanogaster. Adv. Exp. Med. Biol. 332:165-171.

76. Pearlstone, J.R., P. Johnson, M.R. Carpenter, and L.B. Smillie. 1977. Primary structure of rabbit skeletal muscle troponin-T. Sequence determination of the $\mathrm{NH}_{2}$-terminal fragment $\mathrm{CB} 3$ and the complete sequence of troponinT. J. Biol. Chem. 252:983-989.

77. Bullard, B., K. Leonard, A. Larkins, G. Butcher, C. Karlik, and E. Fyrberg. 1988. Troponin of asynchronous flight muscle. J. Mol. Biol. 204:621-637. 